\title{
El tratamiento con laxantes en niños con encopresis sin constipación provocó mayor número de recaidas
}

Lack of benefit of laxatives as adjunctive therapy for functional nonretentive fecal soiling in children. Ginkel R., Benninga M., Blommaart P. y col. J. Pediatr 2000; 137: 808 - 13.

\section{Objetivo}

Comparar los efectos del tratamiento con laxantes y biofeedback vs. biofeedback como único tratamiento en niños encopréticos sin constipación.

\section{Diseño}

Estudio prospectivo en 48 niños que fueron randomizados en dos grupos durante un período estipulado de 7 semanas seguido de un período de seguimiento de 6,12 y 26 semanas hasta 12 meses.

\section{Lugar}

División de Gastroenterología y Nutrición, Departamento de Gastroenterología y Pediatría,Academic Medical Center Amsterdan; y el Departamento de Pediatría del Hospital Sophia Children de Rotterdam, The Netherlands.

\section{Pacientes}

Entre Febrero de 1994 y mayo de 1998, un total de 48 niños (varones $=41$, mujeres $=7$ ) que presentaban encopresis (escurrimiento de materia fecal en la ropa interior en mayores de 4 años) sin síntomas de constipación (definida como menos de 3 movimientos intestinales por semana, pasaje periódico de grandes cantidades de materia fecal, masa palpable abdominal o rectal), edad media de 8 años (rango 5-17), número de encopresis (rango de 2 - 25 por semana)

Intervención

Se randomizó a los niños en dos grupos al azar; uno recibió $5 \mathrm{~g}$ de lactulosa/ $\mathrm{kg}$ de peso cada 12 horas y 5 sesiones de biofeedback de 45 minutos de duración por 7 semanas ( $n=23$ ) y otro grupo solo recibió tratamiento con biofeedback en iguales condiciones sin recibir placebo $(n=25)$

\section{Resultados principales}

Al ingreso se midieron los episodios de encopresis en ambos grupos: pacientes que recibieron laxantes y biofeedback 7 episodios (rango 2-24), pacientes que recibieron biofeedback solo 7 (rango 3-25). Al final del período de intervención, el número de escurrimientos se redujo significativamente en ambos grupos $(p<0.05), 2$ episodios $(0-17)$ y 2 episodios $(0-14)$ respectivamente.

El porcentaje de éxito (menos de 1 episodio de encopresis por semana) fue mayor en el grupo de pacientes que recibió biofeedback (44\%) comparado con el grupo que recibió laxantes (11\%). Asimismo, las recaídas ocurrieron en el $36 \%$ y en el $100 \%$ respectivamente en un período de 6 a 12 meses postintervención.

\section{Conclusiones}

El número de escurrimientos en los pacientes encopréticos sin signos de constipación disminuyó para ambos grupos. El éxito fue mayor con menores recaídas en el grupo que recibió biofeedback solo. No se observaron beneficios adicionales en los niños tratados con laxantes aunque si un mayor número de recaídas. Esto sugiere que estos niños encopréticos deben ser tratados de manera diferente que la encopresis asociada a constipación.

\section{Comentario}

La encopresis, falta de control esfinteriano que provoca manchas de materia fecal en la ropa interior, es un síntoma común de la constipación funcional'1 . El tratamiento se basa en la desimpactación del bolo fecal, el tratamiento prolongado con ablandadores de materia fecal (P. ej.: laxantes) y la reeducación del intestino'.

Este estudio muestra la importancia de diferenciar a los pacientes con encopresis que no se acompañan de constipación para tener mayor éxito en el manejo terapéutico y disminuir el número de recaídas.

Estos pacientes presentan una frecuencia defecatoria normal, una función anorectal estudiada por manometría normal que indicarían mecanismos fisiopatológicos diferentes a la encopresis secundaria a la constipación?2.

El menor porcentaje de éxitos en el grupo que recibió laxantes se debería a que estos pacientes no requieren disminuir la consistencia de la materia fecal, ya que produciría mayores escapes.

Es de importancia destacar que el $46 \%$ de los niños con encopresis sin constipación presentaban enuresis tanto nocturna como diurna. A medida que disminuía el número de encopresis durante el período de tratamiento, los episodios de enuresis también disminuyeron en frecuencia.

Estos resultados sugieren una vez más la importancia del tratamiento conductual ya que ofrece un abordaje psicológico con un componente físico, principalmente dado por la manometría anorectal. Esta técnica proporciona información del esfuerzo eficaz durante la defecación y acompañada de la técnica de biofeedback intenta enseñar y explicar al paciente un registro fisiológico del tratamiento que se le realiza, de forma que pueda percatarse e intentar modificarlos bajo control3.

A pesar de los avances realizados en el tratamiento de estos pacientes, en el cual la técnica de biofeedback juega un rol dentro del tratamiento multidiciplinario, se requieren mayores conocimientos físiopatológicos sobre esta entidad clínica de difícil manejo.

\section{Dra. Andrea Guglielmino [ Servicio de Gastroenterología Htal. de Niños R. Gutierrez ]}

\footnotetext{
Referencias

1.Slap G, Brooks J, Schwartz S. When to perform Biopsies of Enlarged Peripheral Lymph Nodes in young patients. JAMA 1984: 252: 1321-6 2. Slap G, Connor J, Wigton R. Validation of a model to identify young patients for Lymph Node Biopsy. JAMA 1986; 255: 2768-73.

3. Laupacis A, Sekar N, Stiell I. Clinical Prediction Rules: A review and suggested modifications of methodological standars. JAMA 1997:277:488-04.

4. McGinn T, Guyatt G, Wyer P. User's Guides to the Medical Literature: How to use articles about Clinical Decision Rules. JAMA 2000; 284: $79-84$.
} 